\title{
Personalidad múltiple: un caso raro en la practica forense.
}

\author{
Multiple personality: a rare case in forensic practise.
}

L. Fombellida Velasco' y J. A. Sánchez Moro²

\section{RESUMEN}

Se describe un caso de trastorno de identidad disociativo (personalidad múltiple) que motivó estudio forense por tener relación directa con el homicidio de un recién nacido.

La personalidad múltiple es un trastorno poco frecuente, y excepcional en el ámbito forense, consistente en la coexistencia en un individuo de dos o más personalidades independientes. El presente trabajo trata de llamar la atención sobre su existencia y su forma de presentarse.

Palabras clave: Trastornos disociativos, Trastorno de identidad disociativo, Personalidad múltiple. Personalidad alternante.

\section{ABSTRACT}

A case of dissociative identity disorder (multiple personality) is described, which motivated forensic study as it was directly related with a homicide of a newborn baby.

Multiple personality is not a very frequent disorder, exceptional in the forensic environment, consisting of the coexistence in one individual of two or more independent personalities. The present paper tries to call attention to its existence and the way it presents.

Key words: Dissociative disorders, Dissociative identity disorder, Multiple personality disorder. Alternating personality.

Correspondencia: Luis Fombellida Velasco. Juzgado de Primera Inst. e Instrucción n¹. C/ Gran Vía 33. SALAMANCA. Mail: Ifombe@teleline.es

1 Médico Forense. Especialista en Medicina Legal y Forense. Doctor en medicina

2 Médico Forense. 


\section{INTRODUCCIÓN}

Los trastornos disociativos consisten en una alteración de las funciones integradoras de la conciencia, la identidad, la memoria y la percepción del entorno (DSM IV). Experiencias que normalmente se experimentan como una situación continua e integrada dentro de los procesos mentales, se aíslan de ellos alterando el sentimiento de continuidad de sí mismo y el recuerdo de hechos de la vida, y originando una serie de fenómenos clínicos y conductuales con alteración de la memoria e identidad.

Los trastornos disociativos son más una alteración de la organización o estructura mental, que de los contenidos en sí, es decir, se trata de una incapacidad para integrar o asociar unos contenidos, no de una alteración de los mismos [I].

El fenómeno disociativo trata, como un mecanismo defensivo parecido a la represión, de mantener determinada información lejos de la conciencia, almacenándose la información disociada de un modo directo, no transformado y recuperándose (al contrario de lo que ocurre en la represión con los sueños) de la misma forma; comprende un periodo de tiempo, y se desencadena como defensa después de algún episodio psicotraumático.

Amnesia disociativa, fuga disociativa, trastorno de identidad disociativo y trastorno de despersonalización representan los principales trastornos de esta psicopatología, cuyo conocimiento se debe en gran parte a los estudios experimentales sobre la hipnosis. Clásicamente estos trastornos estaban incluidos en la histeria; actualmente, en las clasificaciones internacionales aparecen como categorías propias separadas.

La amnesia disociativa se caracteriza por una incapacidad para recordar información personal importante, generalmente de naturaleza traumática o estresante, que es demasiado amplia para ser explicada por el olvido ordinario (DSM IV).

Cuando, en la amnesia disociativa, la disociación es tan amplia y extensa que llega a conseguir que la identidad está fragmentada junto con la memoria, nos encontramos ante un trastorno de identidad disociativo (personalidad múltiple), forma de disociación que fisura el sentimiento de continuidad de sí mismo y el recuerdo de hechos de la vida.

El trastorno de identidad disociativo, denominado Trastorno de personalidad múltiple por CIE 10, se caracteriza por la presencia de uno o más estados de identidad o personalidad, que controlan el comportamiento del individuo de modo recurrente, junto a una incapacidad para recordar información.

En dicho trastorno se quiebra la característica de identidad de la personalidad que hace que nos sintamos siempre el mismo; aparecen dos o más personalidades alternativamente, con o sin conexión entre ellas, y con amnesia recíproca, ignorando cada personalidad a la otra (personalidad alternante).

Cada vez se manifiesta una de las personalidades con sus características específicas. La existencia de una memoria explícita o episódica que implica el recuerdo de las experiencias personales identificadas con uno mismo, y una memoria implícita o semántica en relación con la ejecución de actividades rutinarias (automáticas), explica que se pueda actuar de un modo automático, desprovisto de la autoidentificación explíitita. disociativo:

EI DSM IV especifica los siguientes criterios para el diagnóstico de Trastorno de identidad

A.- Presencia de dos o más identidades o estados de personalidad (cada una con un patrón propio y relativamente persistente de percepción, interacción y concepción del entorno y de sí mismo). 
B.- Al menos dos de estas identidades o estados de personalidad controlan de forma recurrente el comportamiento del individuo.

C.- Incapacidad para recordar información personal importante, que es demasiado amplia para ser explicada por el olvido ordinario.

D.- El trastorno no es debido a los efectos fisiológicos directos de una sustancia (p. ej., comportamiento automático o caótico por intoxicación alcohólica) o a una enfermedad médica (p. ej., crisis parciales complejas).

La prevalencia estimada del trastorno de identidad disociativo es del 3\% al 5\% entre los pacientes psiquiátricos, según las series estudiadas [2], e inferior al $1 \%$ en la población general a lo largo de la vida, si bien ha aumentado el diagnóstico en las últimas décadas, sobre todo en la psiquiatría americana.

El $90 \%$ de los casos corresponde al sexo femenino.

La disociación aparece como una defensa contra algún trauma distanciando a la persona del mismo y retrasando la integración de este en la vida psíquica.

Etiológicamente el origen del trastorno se relaciona con vivencias psicotraumáticas o con conflictos internos intolerables en la infancia (abusos sexuales o malos tratos) que fuerzan a la mente a segregar la información como un mecanismo de defensa, en un intento de mantener el control mental mientras se ha perdido el control físico [3] [4].

Los modelos dinámicos explican el trastorno como un surgir de nuevas personalidades para adaptarse a situaciones conflictivas, creando estructuras alternativas. Para los conductistas se adoptarían nuevas personalidades para evitar castigos o reprobación.

Se manifiesta casi siempre a partir de la adolescencia y raramente se inicia después de los 40 años, existiendo habitualmente un retraso entre el comienzo de los síntomas y el diagnóstico.

Clínicamente se caracteriza por la presencia de una personalidad primordial o primaria que es la personalidad oficial del paciente, la reconocida por la sociedad, y otras personalidades surgidas por la disociación. Pueden coexistir varias personalidades diferentes, Carrasco Gómez apunta hasta cien [5], de tal forma que la personalidad primaria no tiene conciencia ni control del resto de las personalidades, que adquieren roles y conductas diferentes, o puede tener conciencia de ellas, en cierta medida, considerándolas como compañeros o amigos. El paciente puede ser consciente de espacios de tiempo perdidos, de distorsiones vivenciales del tiempo, o de estados confusionales o lagunas mnésicas, y no conocer sus otras actividades.

La fluctuación de la atención, la perplejidad, la presencia de conductas pasivas y explosiones emocionales no son infrecuentes, constituyendo a veces los únicos síntomas encontrados en la valoración del paciente, siendo necesarias varias entrevistas prolongadas para detectar el trastorno.

El paso de una personalidad a otra suele ser rápido, producido ante factores ambientales o desencadenantes. Normalmente no existe interacción entre ellas, aunque unas personalidades pueden soportar las influencias de otras por impulsos dinámicos internos, por lo que la conducta de la personalidad primaria puede verse motivada o impuesta por otras personalidades que influyen en su acción.

No existen en el Trastorno de identidad disociativo alteraciones de los parámetros biológicos. 


\section{PRESENTACIÓN DEL CASO}

En un contenedor de basura, es encontrado por los basureros una bolsa deportiva conteniendo el cadáver de un recién nacido. La autopsia revela que se trata de un recién nacido vivo a término, de sexo femenino, con signos de vitalidad post-parto, con el cordón umbilical arrancado y sin otros signos de violencia; la data de la muerte se estima en cuarenta y ocho horas y la muerte se considera producida por la anoxia y la acción del frío.

Días después es detenida por la policía una mujer de 27 años de edad, a quien mediante estudio clínico-analítico-ecográfico se le diagnostica un estado puerperal de menos de 10 días de evolución.

La detenida se sorprende de los resultados, negando la evidencia de parto reciente. Su pareja desconoce el estado de embarazo previo.

La historia clínica de la mujer pone de relieve una infancia y adolescencia en un hogar con un padre alcohólico, habiendo presenciado y sufrido muchas vejaciones y malos tratos.

Es madre soltera a los 23 años, con gran angustia y rechazo durante todo el embarazo. Un segundo embarazo año y medio después del primero es vivido de nuevo con angustia, culpa, rechazo y ocultación y el parto tiene lugar en casa en solitario. Posteriormente el niño es dado en adopción.

El embarazo (el tercero) cuyo resultado motiva los hechos judiciales, es fruto de su relación con su actual pareja estable y desencadena los mismos sentimientos que los anteriores. Se lo niega a ella misma; se da cuenta que algo se mueve dentro de ella, pero enseguida lo olvida; se lo oculta a los demás incluso a su pareja (no había engordado demasiado).

Además surgen ideas depresivas y de suicidio. A medida que avanza el embarazo son frecuentes los episodios en los que no puede recordar cosas. Se da cuenta de que su vientre va creciendo, pero no quiere aceptarlo, ni asumirlo. Tiene la sensación de que al darle a una tecla cambia, y empieza a sentirse preocupada, temerosa, angustiosa, por el embarazo, pero después sigue la vida como si no estuviera embarazada.

Es detenida por la policía varios días después de encontrado el recién nacido en la basura; la joven sabía de que se trataba porque lo había visto en la TV y en la prensa, pero no sabía que era ella la autora. Cuando la policía le enseñó la bolsa deportiva en que apareció el recién nacido en el contenedor, la identificó rápidamente y dijo: "yo tengo una igual".

En definitiva negaba absolutamente que en los días anteriores hubiera tenido un parto y hubiera arrojado el producto del parto a un contenedor de basura.

Se realizó la siguiente metódica médico-forense:

Exploración ginecológica y ecográfica, con los siguientes hallazgos: un periné flácido, y violáceo, sin desgarros. Cuello uterino de multípara, permeable a I dedo con secreción sero-sanguinolenta (loquios). Útero aumentado de tamaño, como de 12 semanas ( unos $12 \mathrm{~cm}$.). Areolas mamarias grandes y muy pigmentadas, de las cuales sale un chorro de secreción láctea a la presión.

Se realiza un microlegrado endometrial para estudio histológico (se obtuvo un resultado positivo de células deciduales).

En ecográfica se evidencian las siguientes medidas uterinas: $11,50 \mathrm{~cm}$. de longitud, 7,7 cm. de diámetro antero-posterior. Presencia de pequeños restos (loquios) intraútero.

Estudio de perfil genético de A.D.N. que posteriormente confirma la maternidad de la detenida respecto del recién nacido.

Exploración psicopatológica se encuentra consciente, lúcida, orientada en el tiempo, en el espacio y en la persona, colaborando correctamente en las entrevistas. Durante esta describe múl- 
tiples ocasiones compatibles con estados crepusculares o estrechamiento del campo de la conciencia que cursan con amnesia posterior con preservación parcial de la vigila y sensación de perplejidad "como si estuviera en un escenario y estuviera representando tres papeles a la vez" "como si viviera un flash de repente", y con realización de conductas que solo recuerda al volver a dicho estado.

Atención y concentración: normales.

Afectividad: presenta un estado de ánimo depresivo, con sentimientos de infelicidad, ideas de culpa, baja autoestima, pensamientos negativos. Ideas suicidas. Llanto frecuente durante las entrevistas, con descargas emocionales sobre todo al hablar de sus padres y de los hechos.. Insomnio. Ansiedad asociada con angustia que se exacerba al hablar de los hechos.

Memoria: la memoria próxima se encuentra dentro de la normalidad. La memoria remota se encuentra afectada por amnesias lacunares que se repiten para información personal, sobre todo en relación con sus situaciones de embarazo, con cierta selectividad por los temas del embarazo.

Pensamiento: el curso del pensamiento es normal, con un discurso y un lenguaje lógico, fluido y armónico. El contenido del pensamiento es normal, siendo un pensamiento realista, con alguna idea autorreferencial pero que no presenta francas ideas delirantes ni deliroides.

Sensopercepción: no presenta alteración alguna.

El nivel intelectual se encuentra dentro de los límites convencionales de la normalidad.

Otras pruebas complementarias: se le practica un electroencefalograma (EEG) resultando un trazado sin hallazgos patológicos.

Se le practica el test MMPI-2 obteniéndose un perfil con puntuaciones altas en la escala Pa (paranoia), Pd (desviación psicopática), Pt (psicastenia), y Sc (esquizofrenia).

Se emiten los juicios clínicos de trastorno de identidad disociativo (personalidad múltiple) y trastorno del ánimo de tipo depresivo.

Se informa sobre la inimputabilidad total de la procesada.

La Audiencia Provincial la condena por un delito de homicidio con agravante de parentesco y atenuante de enajenación mental incompleta a seis años de prisión a sustituir, durante igual tiempo, por tratamiento psiquiátrico en régimen ambulatorio.

\section{COMENTARIOS Y VALORACIÓN MÉDICO-FORENSE}

Es evidente que la personalidad múltiple es un trastorno raro, poco diagnosticado o mal conocido, e incluso en algunos medios psiquiátricos hay escepticismo respecto a su existencia [6], afirmando que dicho trastorno es un artefacto o el resultado de una acción del medio debido a modas de novelas o películas (Las tres caras de Eva).

De hecho en la bibliografía de psiquiatría forense al uso, generalmente no se le dedica más de una página, haciendo hincapié en lo raro o poco frecuente de la personalidad múltiple, y la excepcionalidad delictiva [7].

En el presente caso evidenciamos que la paciente ha presentado diversos episodios con un vacío de memoria de un tiempo indeterminado, olvidándose de algunos hechos, pero no todos; generalmente, según la literatura, el período de tiempo olvidado está claramente delimitado. La mayoría de los pacientes son conscientes de haber "perdido parte del tiempo", pero otros tienen "amnesia de la amnesia" y empiezan a ser conscientes del tiempo perdido solamente cuando se enfrentan a la evidencia de que han hecho cosas que no recuerdan, como en nuestro caso que la procesada niega en un principio la comisión de los hechos, niega su parto incluso ante la evidencia de su estado puerperal. La disociación es intensa hasta el grado de determinar la transformación de la personalidad, siendo evidente en la paciente al menos dos personalidades diferentes. 
Además en relación con su situación de embarazo, no es capaz de asumirlo, es algo que la sobrepasa, presentando un trastorno del ánimo de tipo depresivo que es más evidente en una de sus personalidades.

No fue fácil llegar al diagnóstico, tomándose en consideración otras entidades nosológicas como esquizofrenia, trastorno borderline de personalidad, y sobre todo simulación, pues estábamos ante un caso de homicidio de un recién nacido.

Margaret G. Y Spinelli M G. [8] ponen de relieve la frecuencia de la relación entre la muerte del recién nacido de forma violenta el día de su nacimiento con el hecho de la negación previa del embarazo y la disociación.

La propia sintomatología del trastorno de identidad disociativo ofrece el diagnóstico diferencial con las dos primeras (en la esquizofrenia, si existen varias personalidades se manifiestan a la vez). La persistencia sintomatológica durante largas y numerosas entrevistas con la paciente, nos hizo rechazar la simulación [9].

La situación procesal de la enferma, el tratamiento psicofarmacológico a que fue sometida, y su situación personal en dicho módulo penitenciario hospitalario, hacen que poco a poco vaya tomando conciencia, como piezas de un puzzle que van encajando, de la existencia de su enfermedad, de la magnitud de sus actos, revelándonos progresivamente en cada entrevista y exploración la sintomatología disociativa, y poniendo de manifiesto su personalidad múltiple.

La historia biográfica (en especial la existencia de vivencias traumáticas infantiles, como documenta la bibliografía), y la inexistencia de otra patología psíquica compatible y de hallazgos biológicos perfilaron el diagnóstico.

Algunos autores proponen utilizar instrumentos diagnósticos específicos como escalas estructuradas o cuestionarios como el Interviú Clínico Estructurado para Trastornos Disociativos según el DSM-IV (SCID -D o Structured Clinical Interview) de Steinberg, Rounsaville y Cichetti o el Inventario para el Interviú de Trastornos Disociativos (DDIS o Dissociative Disorders Interview Schedule) de Ross, Heber, Norton y Anderson, o la Escala de Experiencias Disociativas de Bernstein y Putnam 1986 (BERNSTEIN E., 1986) (EED, validación española Icarán/Colom/Orengo (ICARAN et al., 1996)), principalmente a utilizar en casos forenses para presentar ante los Tribunales [I0], [I I], [I2].

Para la valoración médico-forense del trastorno de personalidad múltiple realizamos el estudio de la psicogénesis delictiva según criterios fenomenológicos, es decir interpretando las vivencias que la explorada nos manifiesta [13].

Aunque no estamos ante una persona psicótica en el sentido de la nosología psiquiátrica tradicional podemos asimilar el homicidio del recién nacido como un crimen psicótico: impredecible, que sorprende a quien rodea a la autora, sin motivación y sin finalidad racional [14]. En el momento de cometerse se produce también una falta de resonancia emocional o indiferencia, aunque cuando la paciente va tomando conciencia de sus hechos surge un estado emocional depresivo.

En el momento de los hechos, es decir, durante un tiempo indeterminado en relación con el parto, así como en otros periodos posteriores en relación con él, la actividad psíquica y motriz de la explorada estaba regida "por su otra personalidad" de forma que ella no era consciente de sus hechos, la capacidad cognoscitiva estaba anulada; pero no solo esta, sino también la actividad voluntaria (el paso a la acción) estaba anulada también, liberándose conductas automáticas no regidas por el control voluntario de la personalidad.

Durante los lapsus de tiempo en que predomina "la otra personalidad" se dan circunstan- 
cias similares a los estados crepusculares, en los que la conciencia de uno mismo y del mundo exterior está comprometida, al igual que el autocontrol.

Indemnidad en la capacidad cognoscitiva y volitiva de la persona son premisas indispensables para poderle ser atribuidas sus acciones; en nuestro caso no existe en la paciente capacidad de valorar los hechos conforme a derecho ni para actuar en base a esa valoración. Por tanto, faltando aquellas capacidades, la persona que comete un delito en el curso de un trastorno de identidad disociativo no puede ser motivada por la norma jurídica, estando exenta de responsabilidad criminal.

Se recomienda un tratamiento psiquiátrico de tipo farmacológico antidepresivo, y de tipo psicoterápico, tratamiento que puede llevarse a cabo de forma ambulatoria.

\section{BIBLIOGRAFIA}

I.- Hales R. E, Yudofsky S. C, Talbott J. A. Tratado de psiquiatría American Psychiatric Press Edit. Ancora S.A. $2^{\mathrm{a}}$ ed. Barcelona 1996. 669-686

2.- Leal Cercós C. y Leal Cercós M.I.: Histeria. En Trastornos neuróticos. Sociedad española de psiquiatría y Sociedad española de psiquiatría biológica. Psiquiatría editores S.L. Barcelona, 2001. 363-383.

3.- Lewis D. O, Yeager C. A, Swica Y, Pincus J. H, Lewis M. Objective documentation of child abuse and dissociation in 12 murderers with dissociative identity disorder. Am. J. Psychiatry 1997 Dec;154(12):1703-10.

4.-Middleton W, Butler J. Dissociative identity disorder: an Australian series. Aust. N. Z. J. Psychiatry 1998 Dec;32(6):794-804.

5.- Carrasco Gómez]. J. y Maza Martín J. M. Psiquiatría Legal y Forense. Edit. La ley-Actualidad. Madrid. dic. 1977. pp III.37-4 III.37-7.

6.- Sarbin Tr. Múltiple personality disorder: fact 0 artifact? Curr Opin Psychiatry 1997; 10: 136-140.

7.- Gisbert Calabuig J. A. y Sánchez Blanque A.: Neurosis. En Medicina legal y Toxicología. Edit Masson, S.A. $5^{a}$ ed. Barcelona 1988. pp 1035-1051.

8.- Margaret G, Spinelli M G: A systematic investigation of 16 cases of neonaticide. Am. J. Psychiatry May 2001; 158:811-813.

9.- Kaplan H. I, Sadock B. J, Grebb J. A. Sinopsis de psiquiatría. Edit. Médica panamericana S.A. Madrid, 1996. pp649-663.

10.- Steinberg M, Bancroft J, Buchanan J. Multiple personality disorder in criminal law. Bull. Am. Acad. Psychiatry Law 1993;21(3):345-56

II.- Orengo Garćía F. Interpsiquis 2000. Psiquiatría.com.

I2.- Leonard K. N, Telch M. J, Harrington P. J. Dissociation in the laboratory: a comparison of strategies. Behav. Res. Ther. 1999 Jan;37(I):49-61

13.- Bonnet E. F. P. Psicopatología y psiquiatría forenses. López Libreros editores. Buenos Aires (Argentina) 1983.

14.- García Andrade J. A. Psiquiatría criminal y forense. Edit. Centro de estudios Ramón Areces. Madrid 1993. 